Supporting Information

\title{
Strategy of Fusion Covalent Organic Frameworks and Molecularly Imprinted Polymers: A Surprising Effect in Recognition and Loading of Cyanidin-3-O-glucoside
}

Qianyu Zhao,,$^{\dagger}$ Hua Zhang, ${ }^{\dagger}$ Haitian Zhao, ${ }^{\dagger}$ Jia Liu, ${ }^{\S}$ Jingyi Liu,,$^{\dagger}$ Zilong Chen, ${ }^{\ddagger}$ Bin

Li, ${ }^{*}$ Xiaojun Liao, " Joe Mac Regenstein, ${ }^{\perp}$ Jing Wang ${ }^{\#}$ and Xin Yang ${ }^{*},{ }^{+}$

'School of Chemistry and Chemical Engineering, Harbin Institute of Technology, Harbin, Heilongjiang 150001, People's Republic of China

${ }^{7}$ School of Pharmacy, Jiangxi University of Traditional Chinese Medicine, Nanchang, Jiangxi 330004, People's Republic of China

${ }^{s}$ Internal Trade Food Science and Technology Company, Limited, Beijing 102209, People's Republic of China

"National Engineering Research Center for Fruit and Vegetable Processing, College of Food Science and Nutritional Engineering, China Agricultural University, Beijing 100083, People's Republic of China

${ }^{\perp}$ Department of Food Science, Cornell University, Ithaca, New York 14850-7201, United States

\# Key Laboratory of Agro-Product Quality and Safety, Institute of Quality Standard and Testing Technology for Agro-Product, Chinese Academy of Agricultural Sciences, Beijing 100081, People's Republic of China

*Email: yangxin@hit.edu.cn

Table of Contents (28 Pages) 
Section A. Supporting experimental section:

(Pages S3)

Section B. Supporting Schemes:

(Pages S4-S5)

Scheme S1----Scheme S2

Section C. Supporting Figures:

(Pages S6-S19)

Figure S1----Figure S14

Section D. Supporting Tables:

(Pages S20-S25)

Table S1----Table S6

Section E. Reference

(Pages S26-

S27) 


\section{Section A. Supporting experimental section:}

\section{Preparation methods for anthocyanins extract:}

Fresh plants were pulped at $4{ }^{\circ} \mathrm{C}$, weighed $10.0 \mathrm{~g}$, and the ratio of material to liquid was 1:5. Ultrasonic extraction was carried out 3 times for 20 minutes at room temperature in the dark with $70 \%$ ethanol solution (containing $0.1 \%$ hydrochloric acid). The filtrate was obtained by vacuum filtration through a Buchner funnel, and concentrated under vacuum at $45{ }^{\circ} \mathrm{C}$ to remove ethanol. After freeze-drying, anthocyanin extract was obtained. 
Section B. Supporting Schemes:

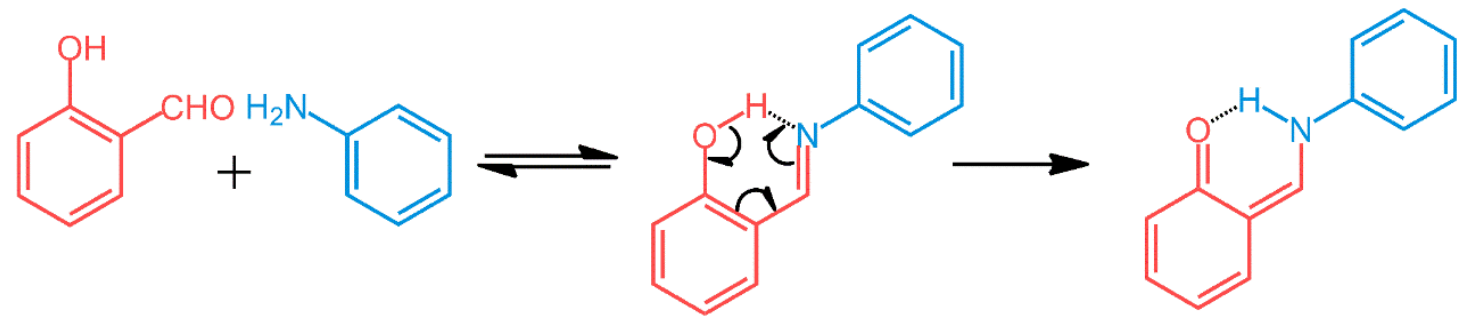

Scheme S1. Reaction equation for the exchange of enol and keto form ${ }^{1}$. 


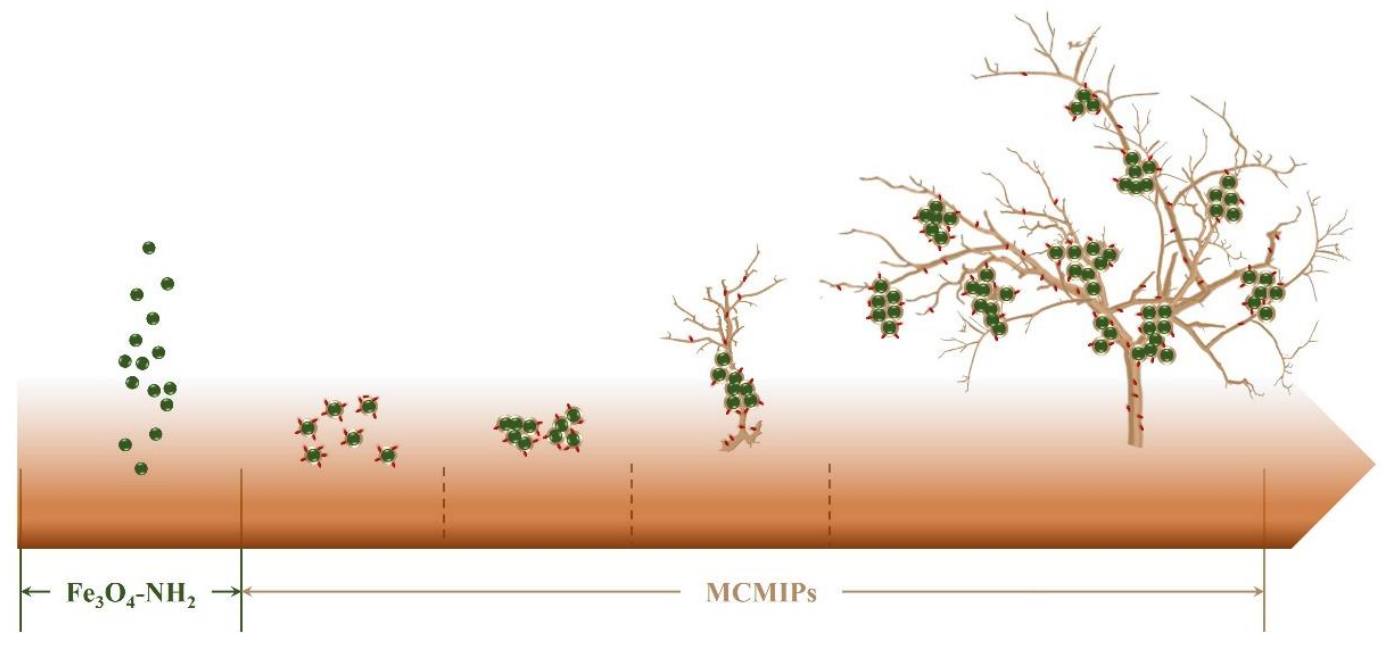

Scheme S2. Illustration of the growth trend of MCMIPs. 


\section{Section C. Supporting Figures:}

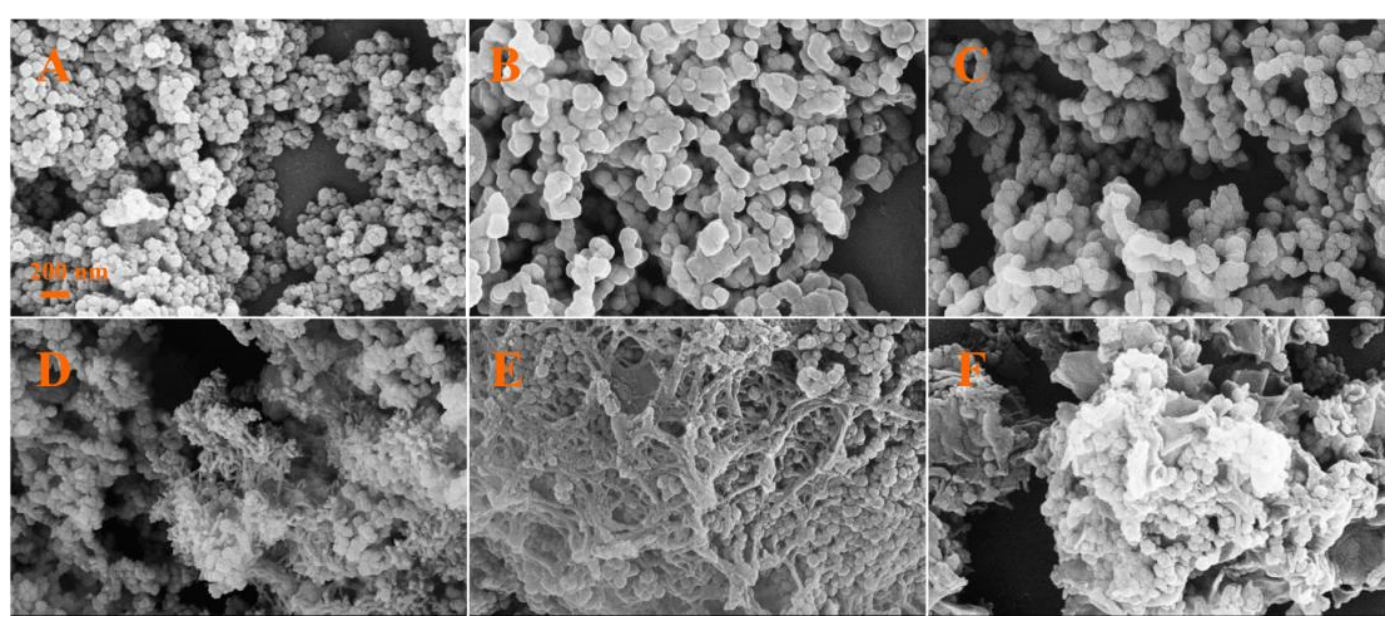

Figure S1. SEM images of MCMIPs composed of different functional monomers (A:

DADHAQ, B: ODA, C: BD, D: Pa-1, E: DHBD and F: DT). 


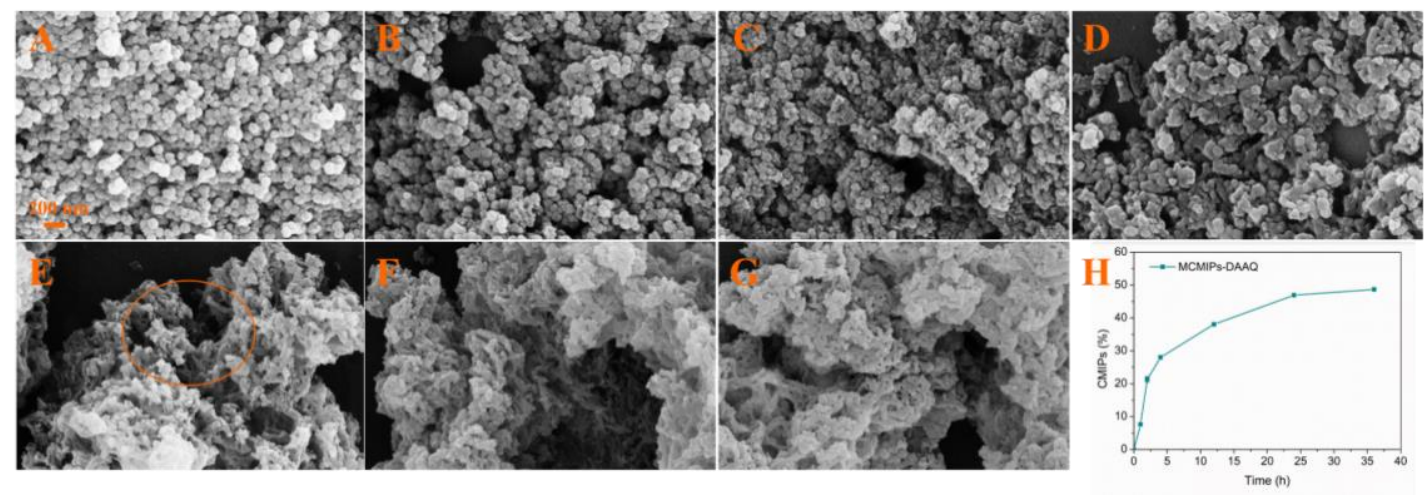

Figure S2. SEM images of different formation times of MCMIPs-DAAQ (A: 0 h, B:

$1 \mathrm{~h}, \mathrm{C}: 2 \mathrm{~h}, \mathrm{D}: 4 \mathrm{~h}, \mathrm{E}: 12 \mathrm{~h}, \mathrm{~F}: 24 \mathrm{~h}$ and G: $36 \mathrm{~h})$ and (H) the mass fraction of CMIPsDAAQ at different formation times. 
A
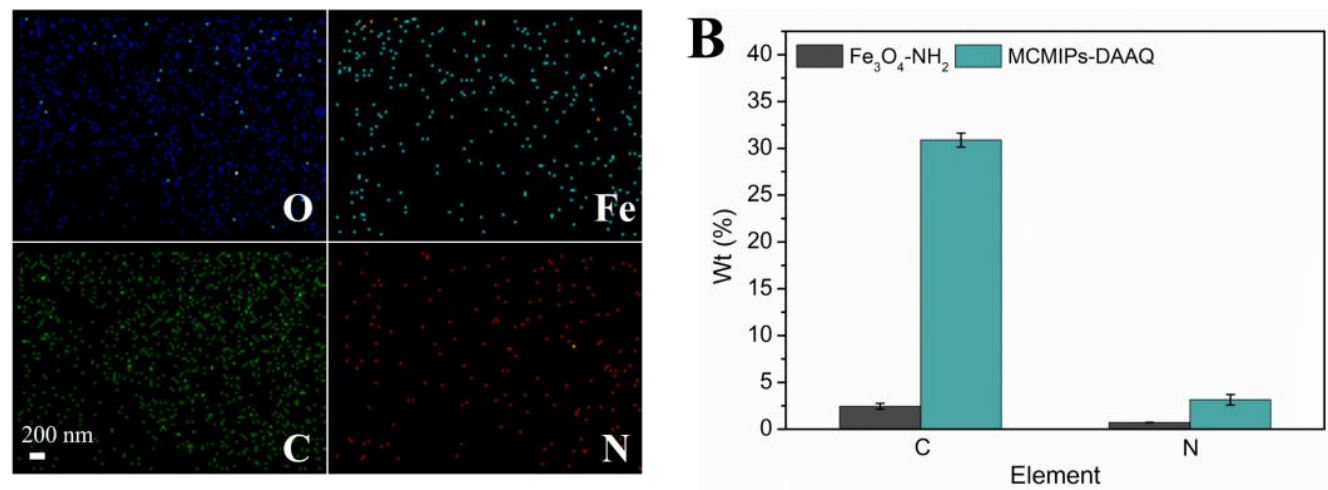

Figure S3. (A) elemental mappings of C, N, O, and Fe atoms from the MCMIPs-

DAAQ, (B) elemental analysis. 


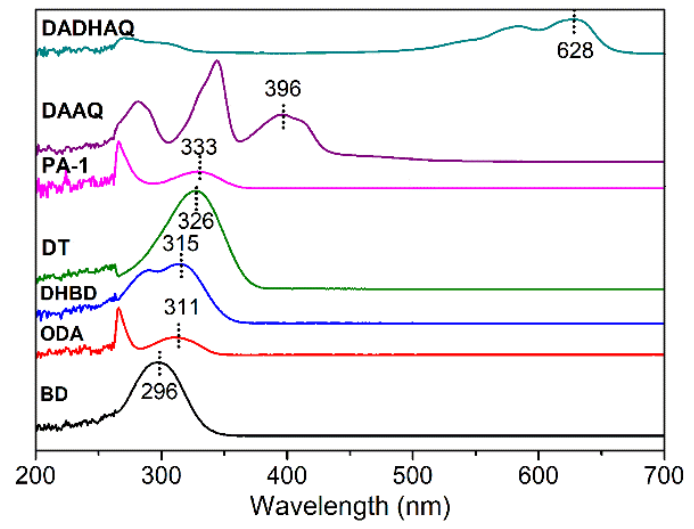

Figure S4. UV-Vis spectrums of functional monomers. 

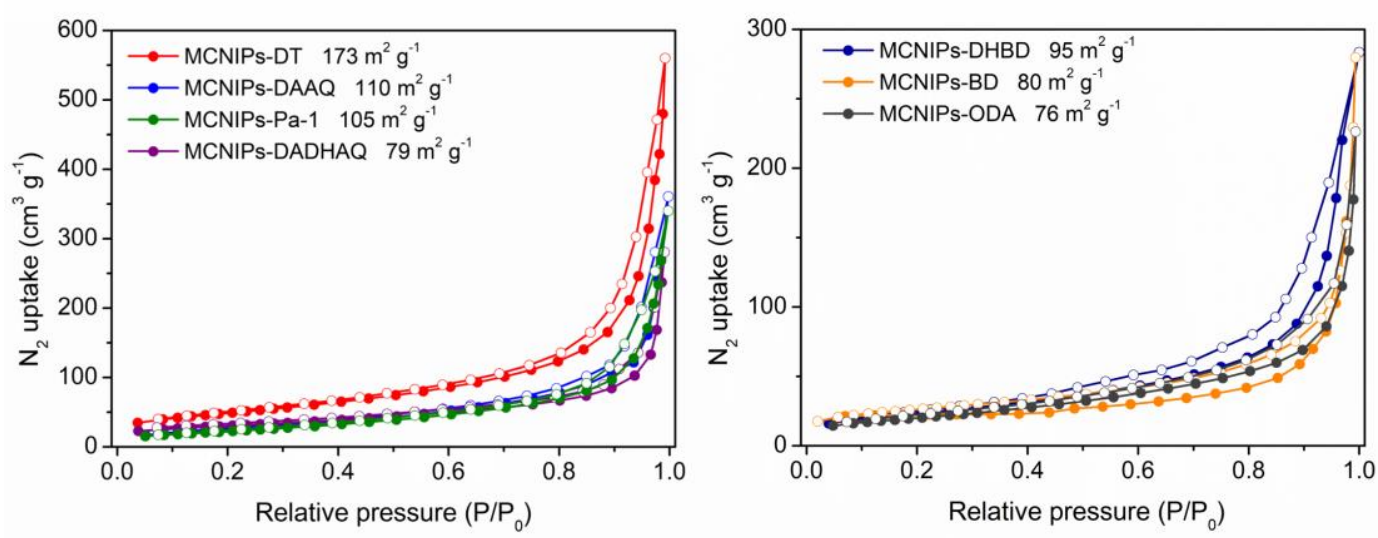

Figure S5. $\mathrm{N}_{2}$ adsorption isotherms at $77 \mathrm{~K}$ of MCNIPs composed of different functional monomer. 


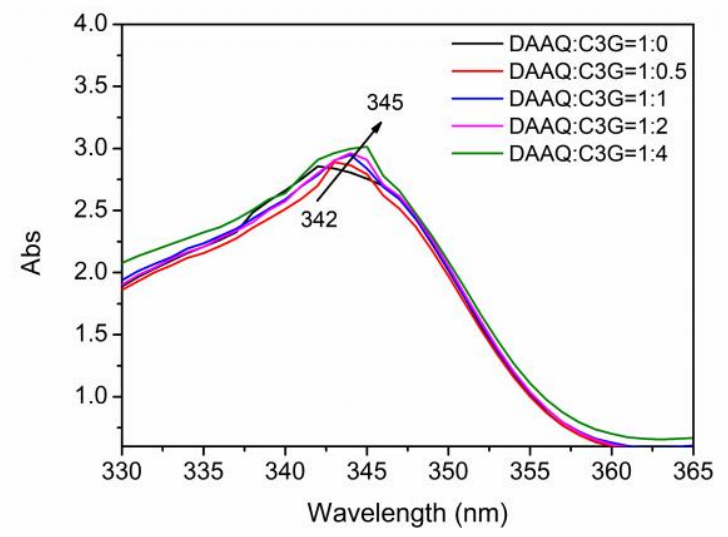

Figure S6. UV-vis absorption spectra of DAAQ with different amounts of C3G

$$
\left(\mathrm{C}_{\mathrm{DAAQ}}=0.08 \mathrm{mmol} \mathrm{L}^{-1}\right)
$$




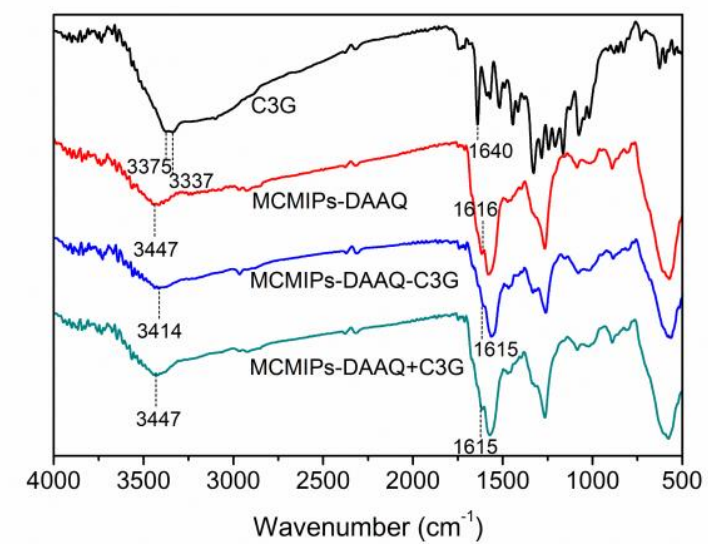

Figure S7. FT-IR spectra of C3G, MCMIPs-DAAQ, MCMIPs-DAAQ after adsorption of C3G (MCMIPs-DAAQ-C3G), the physical mixture of MCMIPs-DAAQ and C3G (MCMIPs-DAAQ + C3G). 


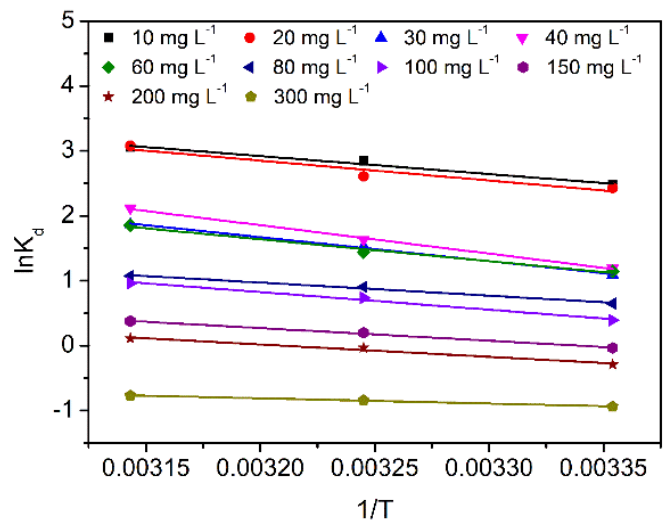

Figure S8. Effect of temperature on the sorption of C3G on MCMIPs-DAAQ

(shaking time: $240 \mathrm{~min}, \mathrm{pH}=7$, adsorption dose: $\mathrm{V}=5 \mathrm{~mL}, \mathrm{~m}=5 \mathrm{mg}$ of MCMIPs-

DAAQ at different concentration). 

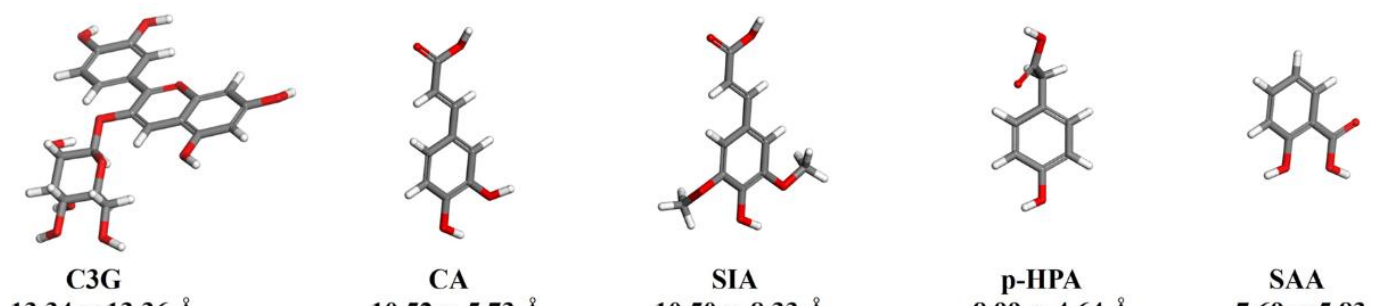

$13.34 \times 13.36 \AA$
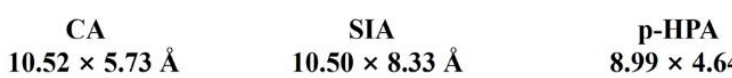

p-HPA
$8.99 \times 4.64 \AA$

SAA

$10.50 \times 8.33 \AA$
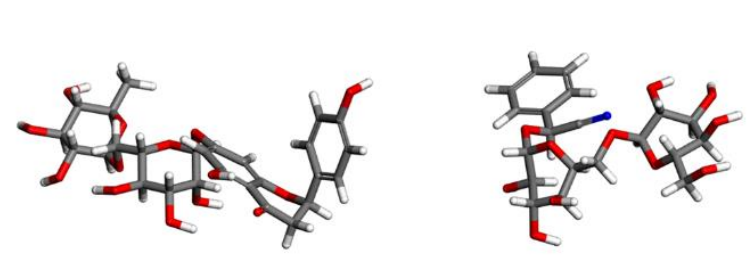

$\mathrm{Ru}$

Qu

$13.10 \times 7.59 \AA$

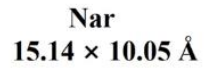

Amy

$19.53 \times 4.64 \AA$

Figure S9. Structure of analogues. White: hydrogen, gray: carbon, red: oxygen, blue:

nitrogen. 


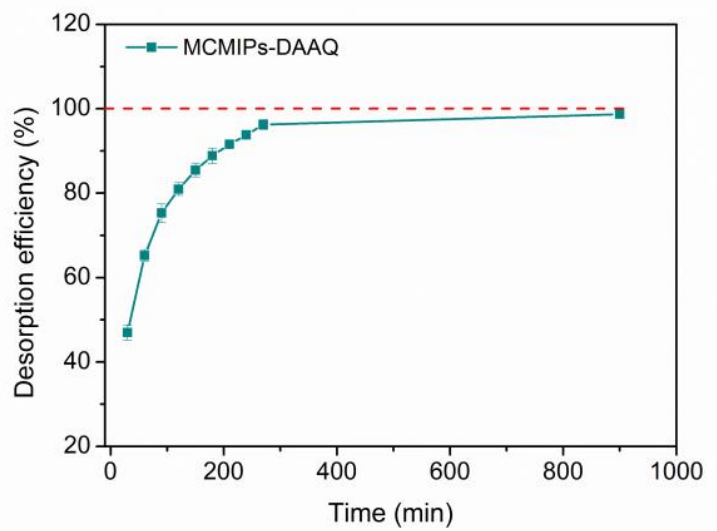

Figure S10. Elution curve of MCMIPs-DAAQ. 


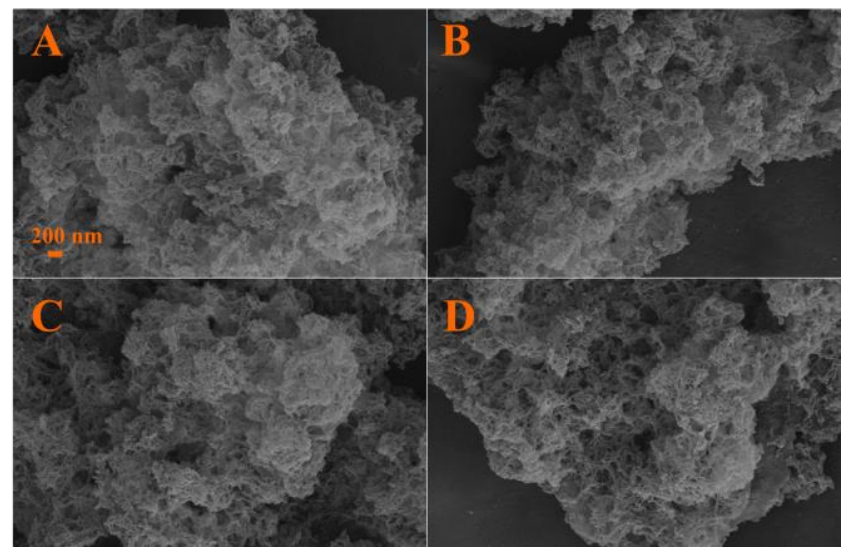

Figure S11. SEM images of MCMIPs-DAAQ after $\mathrm{n}$ adsorption-desorption cycles (A: $n=0, B: n=3, C: n=6, D: n=10)$. 


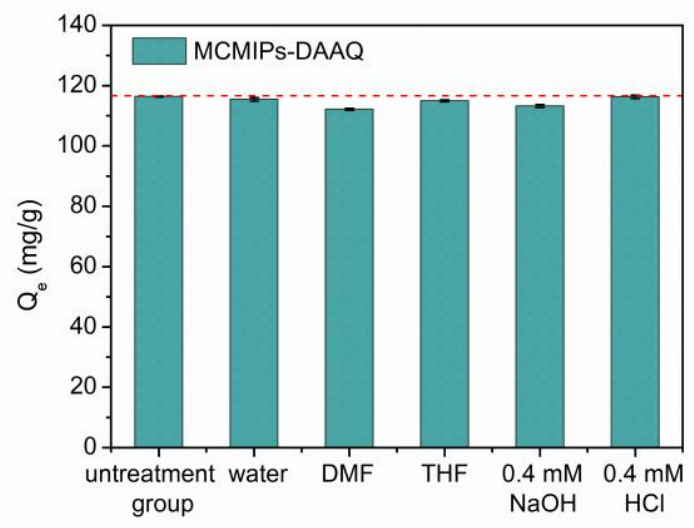

Figure S12. Adsorption of $\mathrm{C} 3 \mathrm{G}$ on MCMIPs-DAAQ after treatment with different reagents for $24 \mathrm{~h}$. 


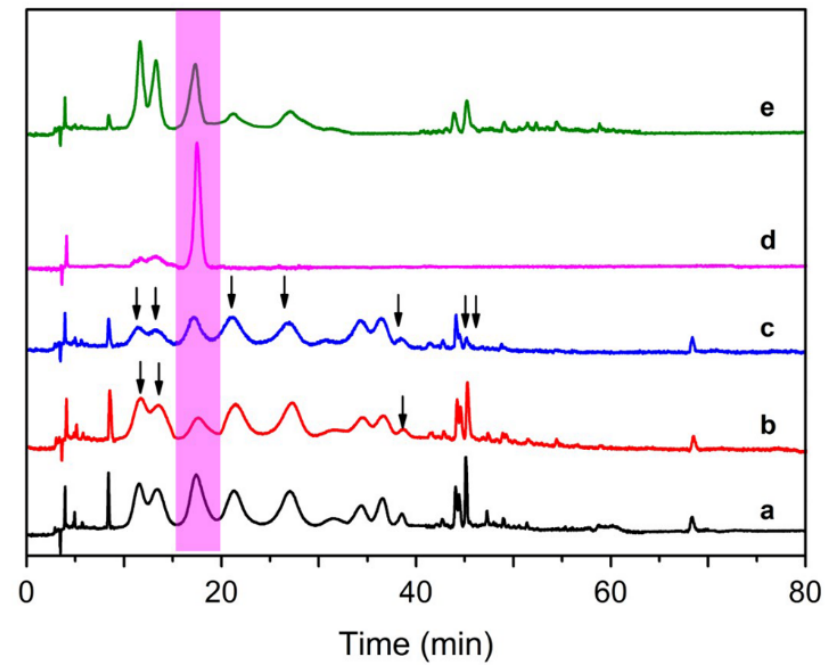

Figure S13. Chromatograms of whortleberry extract, (a) initial sample, (b) the sample after extraction by MCMIPs-DAAQ, (c) the sample after extraction by MCNIPsDAAQ, (d) elution from MCMIPs-DAAQ and (e) elution from MCNIPs-DAAQ.

Pink band: $\mathrm{C} 3 \mathrm{G}$. 


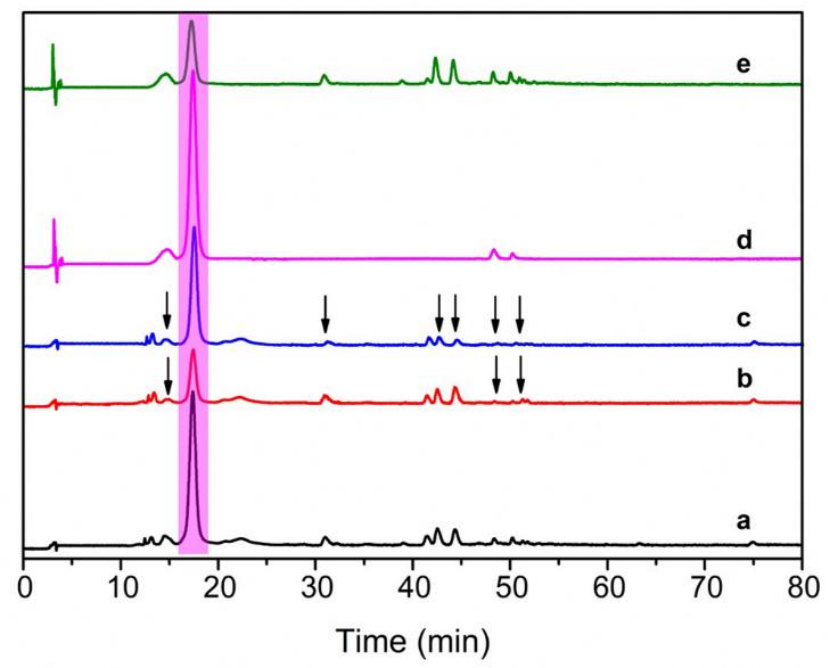

Figure S14. Chromatograms of blueberry extract, (a) initial sample, (b) the sample after extraction by MCMIPs-DAAQ, (c) the sample after extraction by MCNIPsDAAQ, (d) elution from MCMIPs-DAAQ and (e) elution from MCNIPs-DAAQ.

Pink band: $\mathrm{C} 3 \mathrm{G}$. 
Section D. Supporting Tables:

Table S1. Adsorption isotherm parameters of C3G on MCMIPs-DAAQ.

\begin{tabular}{|c|c|c|c|c|c|c|c|}
\hline \multirow[b]{2}{*}{$\begin{array}{c}\text { Temperature } \\
(\mathrm{K})\end{array}$} & \multirow[b]{2}{*}{$\begin{array}{c}Q_{e} \\
\left(\mathrm{mg} \mathrm{g}^{-1}\right)\end{array}$} & \multicolumn{3}{|c|}{$\begin{array}{c}\text { Langmuir isotherm } \\
\text { model }\end{array}$} & \multicolumn{3}{|c|}{$\begin{array}{c}\text { Freundlich isotherm } \\
\text { model }\end{array}$} \\
\hline & & $\begin{array}{c}Q_{\max } \\
\left(\mathrm{mg} \mathrm{g}^{-1}\right) \\
\end{array}$ & $K_{L}$ & $R^{2}$ & $K_{F}$ & $\mathrm{n}$ & $R^{2}$ \\
\hline 298.15 & 112.44 & 59.10 & 0.24 & 0.924 & 12.32 & 2.43 & 0.959 \\
\hline 308.15 & 120.13 & 63.73 & 0.31 & 0.944 & 14.66 & 2.44 & 0.967 \\
\hline 318.15 & 126.71 & 72.52 & 0.34 & 0.948 & 17.64 & 2.55 & 0.958 \\
\hline
\end{tabular}


Table S2. Values of various thermodynamic parameters for the adsorption of C3G on MCMIPs-DAAQ.

\begin{tabular}{|c|c|c|c|c|}
\hline$C_{0}\left(\mathrm{mg} \mathrm{L}^{-1}\right)$ & $\Delta H^{0}\left(\mathrm{~kJ} \mathrm{~mol}^{-1}\right)$ & $\Delta S^{0}\left(\mathrm{~J} \mathrm{~K}^{-1} \mathrm{~mol}^{-1}\right)$ & $\mathrm{T}(\mathrm{K})$ & $\Delta G^{0}\left(\mathrm{~kJ} \mathrm{~mol}^{-1}\right)$ \\
\hline \multirow[t]{3}{*}{10} & 23.09 & 98.18 & 298.15 & -6.18 \\
\hline & & & 308.15 & -7.16 \\
\hline & & & 318.15 & -8.15 \\
\hline \multirow[t]{3}{*}{20} & 25.48 & 105.24 & 298.15 & -5.90 \\
\hline & & & 308.15 & -6.95 \\
\hline & & & 318.15 & -8.00 \\
\hline \multirow[t]{3}{*}{30} & 30.75 & 112.29 & 298.15 & -2.73 \\
\hline & & & 308.15 & -3.86 \\
\hline & & & 318.15 & -4.98 \\
\hline \multirow[t]{3}{*}{40} & 36.43 & 132.00 & 298.15 & -2.93 \\
\hline & & & 308.15 & -4.25 \\
\hline & & & 318.15 & -5.57 \\
\hline \multirow[t]{3}{*}{60} & 28.03 & 103.34 & 298.15 & -2.78 \\
\hline & & & 308.15 & -3.81 \\
\hline & & & 318.15 & -4.84 \\
\hline \multirow[t]{3}{*}{80} & 16.79 & 61.82 & 298.15 & -1.64 \\
\hline & & & 308.15 & -2.26 \\
\hline & & & 318.15 & -2.88 \\
\hline \multirow[t]{3}{*}{100} & 22.55 & 79.02 & 298.15 & -1.01 \\
\hline & & & 308.15 & -1.80 \\
\hline & & & 318.15 & -2.59 \\
\hline \multirow[t]{3}{*}{150} & 16.13 & 62.18 & 298.15 & -2.41 \\
\hline & & & 308.15 & -3.03 \\
\hline & & & 318.15 & -3.65 \\
\hline \multirow[t]{3}{*}{200} & 15.84 & 59.16 & 298.15 & -1.80 \\
\hline & & & 308.15 & -2.39 \\
\hline & & & 318.15 & -2.98 \\
\hline \multirow[t]{3}{*}{400} & 6.73 & 23.09 & 298.15 & -0.16 \\
\hline & & & 308.15 & -0.39 \\
\hline & & & 318.15 & -0.62 \\
\hline
\end{tabular}


Table S3. Adsorption kinetics parameters of C3G on MCMIPs-DAAQ.

\begin{tabular}{cccccccc}
\hline & \multicolumn{3}{c}{ Pseudo-first-order model } & & \multicolumn{3}{c}{ Pseudo second order model } \\
\cline { 2 - 3 } \cline { 6 - 8 } $\begin{array}{c}Q_{e} \\
\left(\mathrm{mg} \mathrm{g}^{-1}\right)\end{array}$ & $\begin{array}{c}Q_{1 \text { lcal }} \\
\left(\mathrm{mg} \mathrm{g}^{-1}\right)\end{array}$ & $k_{1}$ & $R^{2}$ & & $\begin{array}{c}Q_{2 c a l} \\
\left(\mathrm{mg} \mathrm{g}^{-1}\right)\end{array}$ & $k_{2}$ & $R^{2}$ \\
\hline 115.52 & 6248.27 & 0.012 & 0.901 & & 117.65 & 0.00056 & 0.999 \\
\hline
\end{tabular}


Table S4. Distribution ratio $\left(K_{d}\right)$, selectivity coefficient $\left(k^{s e l}\right)$ and relative selectively coefficient $\left(k^{r e l}\right)$ values of MCMIPs-DAAQ and MCNIPs-DAAQ for different analytes.

\begin{tabular}{cccccc}
\hline Analytes $^{\mathrm{a}}$ & $\begin{array}{c}K_{d, \text { MCMIPs }}^{\mathrm{b}} \\
(\mathrm{L} / \mathrm{g})\end{array}$ & $k^{\text {sel }}{ }_{\text {MCMIPs }}{ }^{\mathrm{c}}$ & $\begin{array}{c}K_{d, \text { MCNIPs }}{ }^{\mathrm{b}} \\
(\mathrm{L} / \mathrm{g})\end{array}$ & $k^{\text {sel }}{ }_{\text {MCNIPs }}{ }^{\mathrm{c}}$ & $k^{\text {rel, d }}$ \\
\hline C3G & 0.3753 & $/$ & 0.1488 & $/$ & $/$ \\
SAA & 0.0315 & 11.90 & 0.0358 & 4.16 & 2.86 \\
SIA & 0.0247 & 15.19 & 0.0555 & 2.69 & 5.65 \\
CA & 0.0317 & 11.83 & 0.0389 & 3.83 & 3.09 \\
p-PHA & 0.0167 & 22.42 & 0.0211 & 7.06 & 3.17 \\
Nar & 0.0041 & 91.17 & 0.0235 & 6.33 & 14.40 \\
Ru & 0.0604 & 6.21 & 0.0497 & 3.00 & 2.07 \\
Qu & 0.0631 & 5.95 & 0.0531 & 2.80 & 2.12 \\
Amy & 0.0565 & 6.64 & 0.0432 & 3.45 & 1.93 \\
\hline
\end{tabular}

a. $\mathrm{C} 3 \mathrm{G}$ was chosen as a template molecule and other analytes as referent molecules.

b. ${ }^{2}$ Distribution ratio $K_{d}=Q_{e} / C_{e}$.

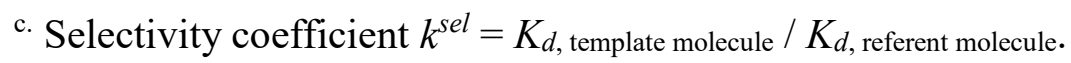

d. Relative selectively coefficient $k^{r e l}=k^{\text {sel }}$ MCMIPs $/ k^{\text {sel }}$ MCNIPs.

Table S5. Distribution ratio $\left(K_{d}\right)$, selectivity coefficient $\left(k^{s e l}\right)$ values of PAM for 
different analytes.

\begin{tabular}{ccc}
\hline Analytes & $K_{d, \text { PAM }}(\mathrm{L} / \mathrm{g})$ & $k^{\text {sel }}$ PAM \\
\hline $\mathrm{C} 3 \mathrm{G}$ & 0.1284 & $/$ \\
$\mathrm{Nar}$ & 0.0468 & 9.16 \\
$\mathrm{Ru}$ & 0.1138 & 2.75 \\
$\mathrm{Qu}$ & 0.0140 & 1.13 \\
\hline
\end{tabular}

Table S6. Comparison of MCMIPs performance with some recently reported sorbents 


\begin{tabular}{|c|c|c|c|c|c|}
\hline Adsorbent & Adsorbate & $\begin{array}{l}\text { Adsorption } \\
\text { capacity } \\
\left(\mathrm{mg} \mathrm{g}^{-1}\right)\end{array}$ & $\begin{array}{c}\text { desorption } \\
\text { ratio }(\%)\end{array}$ & $\begin{array}{l}\text { Adsorption } \\
\text { equilibrium } \\
\text { time (min) }\end{array}$ & Reference \\
\hline $\begin{array}{c}\text { MCMIPs- } \\
\text { DAAQ }\end{array}$ & $\mathrm{C} 3 \mathrm{G}$ & 115.52 & 98.68 & 240 & This work \\
\hline DMMIPs & C3R & 15.69 & l & 60 & 3 \\
\hline Laponite ${ }^{\circledR}$ & $\mathrm{AOC}$ & 14.65 & 84.86 & 15 & 4 \\
\hline $\begin{array}{c}\text { Clay- } \\
\text { polyethyle } \\
\text { ne }\end{array}$ & $\mathrm{AOC}$ & 11.76 & / & I & 5 \\
\hline Bentonite & $\mathrm{AOC}$ & 0.24 & 11.24 & / & \multirow{4}{*}{6} \\
\hline Charcoal & $\mathrm{AOC}$ & 0.12 & 14.68 & I & \\
\hline $\begin{array}{c}\text { Amberite } \\
\text { XAD2 }\end{array}$ & & 0.67 & 14.70 & I & \\
\hline $\begin{array}{c}\text { Amberite } \\
\text { XAD4 }\end{array}$ & & 0.59 & 46.32 & l & \\
\hline XAD-7HP & $\mathrm{AOC}$ & 5.39 & 98.20 & 175 & 7 \\
\hline$X-5$ resin & $\mathrm{AOC}$ & 25.7 & 57.00 & 400 & 8 \\
\hline Oasis HLB & $\mathrm{AOC}$ & 5.76 & 72.48 & I & \multirow{2}{*}{9} \\
\hline $\begin{array}{c}\text { Isolute C8 } \\
\text { (EC) }\end{array}$ & & 3.06 & 69.80 & / & \\
\hline
\end{tabular}

Section E. Reference: 
(1) Kandambeth, S.; Mallick, A.; Lukose, B.; Mane, M. V.; Heine, T.; Banerjee, R. Construction of Crystalline 2D Covalent Organic Frameworks with Remarkable Chemical (Acid/Base) Stability via a Combined Reversible and Irreversible Route. $J$. Am. Chem. Soc. 2012, 134, 19524-19527.

(2) Ji, W.; Sun, R.; Geng, Y.; Liu, W.; Wang, X. Rapid, Low Temperature Synthesis of Molecularly Imprinted Covalent Organic Frameworks for the Highly Selective Extraction of Cyano Pyrethroids from Plant Samples. Anal. Chim. Acta 2018, 1001, 179-188.

(3) Zhao, Q.-Y.; Zhao, H.-T.; Yang, X.; Zhang, H.; Dong, A.-J.; Wang, J.; Li, B. Selective Recognition and Fast Enrichment of Anthocyanins by Dummy Molecularly Imprinted Magnetic Nanoparticles. J. Chromatogr. A 2018, 1572, 9-19.

(4) Capello, C.; Leandro, G. C.; Maduro Campos, C. E.; Hotza, D.; Mattar Carciofi, B. A.; Valencia, G. A. Adsorption and Desorption of Eggplant Peel Anthocyanins on a Synthetic Layered Silicate. J. Food Eng. 2019, 262, 162-169.

(5) Lopes, T. J.; Goncalves, O. H.; Quadri, M. G. N.; Machado, R. A. F.; Quadri, M. B. Adsorption of Anthocyanins using Clay-Polyethylene Nanocomposite Particles. Appl. Clay Sci. 2014, 87, 298-302.

(6) Das, A. B.; Goud, V. V.; Das, C. Adsorption/Desorption, Diffusion, and Thermodynamic Properties of Anthocyanin from Purple Rice Bran Extract on Various Adsorbents. J. Food Process Eng. 2018, 41, DOI: 10.1111/jfpe.12834.

(7) Wu, Y.; Han, Y.; Tao, Y.; Fan, S.; Chu, D.-T.; Ye, X.; Ye, M.; Xie, G. Ultrasound Assisted Adsorption and Desorption of Blueberry Anthocyanins using Macroporous 
Resins. Ultrason. Sonochem. 2018, 48, 311-320.

(8) Zhang, P.; Wang, L.; Fang, S. Modeling of the Adsorption/Desorption Characteristics and Properties of Anthocyanins from Extruded Red Cabbage Juice by Macroporous Adsorbent Resin. Int. J. Food Eng. 2019, 15, DOI: 10.1515/ijfe-20180239.

(9) Trikas, E. D.; Papi, R. M.; Kyriakidis, D. A.; Zachariadis, G. A. Evaluation of Ion Exchange and Sorbing Materials for Their Adsorption/Desorption Performane towards Anthocyanins, Total Phenolics, and Sugars from a Grape Pomace Extract. Separations 2017, 4, DOI: 10.3390/separations4010009. 